\title{
Design of Robust Structures Against Unexpected Failures
}

\author{
Tatsuya Iwase* Emmanuel Tromme* Atsushi Kawamoto* \\ * Multiphysics Analysis $\&$ Design Program, Toyota Central RED Labs, \\ Aichi, Japan (e-mail: \{tiwase;etromme;atskwmt\}@mosk.tytlabs.co.jp).
}

Keywords: Topology optimization, robustness, local volume constraint, failure, game theory.

\section{INTRODUCTION}

Enforcing local volume constraints in the design problem turns out to be an efficient approach to increase structural robustness against uncertainties (Wu et al., 2017). By constraining locally the available amount of material, the optimized design exhibits infill patterns which reflects a more uniform and periodic distribution of the material. This method can notably produce results exhibiting bonelike structures which are known to be robust against load uncertainty. Compared to a deterministic approach, the robustness is often achieved at the cost of reducing the component stiffness (Tromme et al., 2017). However, the justification of using local volume constraints has not been well discussed in the context of robust topology optimization. Moreover, no standard methodology exists to determine the local volume constraint upper bound.

This study aims to explain the relationship between robustness and local volume constraints and to propose a method defining the upper bound of local volume constraints for a given failure distribution. To compute the failure probability distribution, a game theory approach is adopted. A standard design problem is solved to illustrate the developed method.

\section{ROBUST TOPOLOGY OPTIMIZATION}

Standard topology optimization problem is usually formulated as a compliance minimization problem (stiffness maximization) subject to a global volume constraint. Mathematically, the formulation reads

$$
\begin{array}{cl}
\underset{\phi}{\operatorname{minimize}} & f_{0}=\mathbf{f}^{T} \mathbf{d} \\
\text { subject to } & \mathbf{K}(\boldsymbol{\phi}) \mathbf{d}=\mathbf{f}, \\
& \sum_{e \in \Omega} \rho_{e}(\boldsymbol{\phi}) v_{e} \leq V, \\
& -1 \leq \phi_{i} \leq 1, \quad \forall i \in \Omega,
\end{array}
$$

where $\Omega$ is the design domain, $\mathbf{K}$ the stiffness matrix, $\mathbf{d}$ the displacement vector and $\mathbf{f}$ the force vector. The element density and the element volume are represented respectively by $\rho_{e}$ and $v_{e}$. The vector $\phi$ gathers the design variables.

Topology optimization problems are known to be ill-posed and prone to checkerboard issues and mesh dependency. In this paper, the Helmholtz PDE based filter is adopted and introduces the relationship between the design variable $\phi$ and the element density $\boldsymbol{\rho}$ (Kawamoto et al., 2011). To mathematically enforce a 0-1 material distribution, the SIMP interpolation scheme is considered (Bendsøe, 1989; Rozvany et al., 1992).

Ensuring robustness via local volume constraints is straightforward as it simply adds a set of constraints to the design problem (1). Those local constraints are expressed as

$$
\sum_{k \in \Omega_{l}} \rho_{k} v_{k} \leq V_{l}, \quad \text { for } l \in \mathcal{L}=\{1 \ldots L\},
$$

where $L$ is the number of design domain subdivisions and $V_{l}$ the upper bound of the subdomain $l$. The subdivision verifies

$$
\bigcup_{l \in \mathcal{L}} \Omega_{l}=\Omega \quad \text { and } \quad \Omega_{i} \cap \Omega_{j}=0 \text {, for } i \neq j .
$$

\section{UNEXPECTED FAILURE}

The present study focuses on design problems considering unexpected failures. It is assumed that failure arises from an unexpected load other than $\mathbf{f}$ and that the probability distribution of the failure does not depend on the structure nor on the load vector. This situation can occur due to defects in production, accidental impacts during transportation or aged deterioration for instance.

The failure distribution is represented by $p_{l}(l \in \mathcal{L})$ with $\sum_{l \in \mathcal{L}} p_{l}=1$. If the structure undergoes a failure in the subdomain $l$, the stiffness drops in that region. Therefore, if an algorithm could predict that the subdomain $l$ will have a failure, it would be advantageous to remove material in $l$ and to redistribute it in other subdomains. Hence, the larger failure probability $p_{l}$ is expected, the smaller amount of material, i.e. the smaller upper bound $V_{l}$, should be applied.

The problem is to compute the upper bound $V_{l}$ based on the given probability distribution $p_{l}$. For this purpose, we assume a design process that reallocates uniformly materials in other subdomains. The following lemma summarizes the main achievement of this research as it gives a relationship between the upper bound of the local volume constraints and the probability distribution.

Lemma 1. If the failure distribution $p_{l}$ is given, the upper bound of the local volume constraint $V_{l}$ can be computed as

$$
V_{l}=\frac{1-p_{l}}{L-1} V, \quad \text { for } l \in \mathcal{L} .
$$


The proof is omitted due to the limited space. However, it can easily be checked that the sum of the local volume constraint upper bounds equals the global volume constraint upper bound, i.e. $\sum_{l \in \mathcal{L}} V_{l}=V$.

\section{GAME THEORY APPROACH}

To determine the failure distribution $p_{l}$, we consider the particular case wherein some opponents try to identify the weakest point of the structure. In such worst case scenarios, game theory can be employed to determine the failure distribution while guaranteeing robustness (Holmberg et al., 2017).

The situation can be compared to a penalty shoot-out in a soccer game. There are two players, a kicker and a keeper. The kicker aims at the areas of the goal where it is difficult for the keeper to reach, i.e. the extreme left or right. However, the keeper tries to predict the best action of the kicker and he concludes that the kicker will aim at the goal extremities, which is not convenient for the kicker as his strategy is leaked. As a result, the options of both players converge towards some compromising points.

Similarly, design problems considering unexpected failure can be interpreted as a game between a "failure" player and a "structure" player. The failure player tries to choose the subdomain $l$ that maximizes the structural damage while the structure player tries to limit the amount of material within the targeted areas. In game theory, this is a standard two player zero-sum game. The utility of the failure player is the compliance of the structure after failure and the utility of the structure player is the opposite. The resulting failure distribution $p_{l}$ can be computed as a mixed Nash equilibrium of the game. The mixed Nash equilibra of two player games can be computed using the Lemke-Hawson algorithm (Papadimitriou, 2007).

\section{EXAMPLE AND CONCLUSION}

The design problem described in Fig. 1 is solved to exemplify the proposed method. The available amount of material is set to $50 \%$ of the design domain which is divided into $L=8 * 16=128$ subdomains. It is assumed that the subdomain loses all its material if a failure occurs.

The failure distribution $p_{l}$ is first computed from the game theory problem. To reduce computation time, the number of subdomains is reduced to eight. The players have six options since the two subdomains where the load is applied need material to withstand the load. The mixed Nash equilibrium computed by Lemke-Hawson algorithm is not uniform as illustrated in Fig. 2, which means that specific subdomains are targeted by the failure player. The local volume constraint $V_{l}$ is then computed based on (4). The optimized solution is illustrated in Fig. 3.

This first example illustrates the validity of the proposed approach. Comparing to a standard optimization, the compliance is higher, meaning that the structure is less stiff but it is however more robust against unexpected failure.

\section{REFERENCES}

Bendsøe, M. (1989). Optimal shape design as a material distribution problem. Structural optimization, 1, 193202. doi:10.1007/s004190050248.

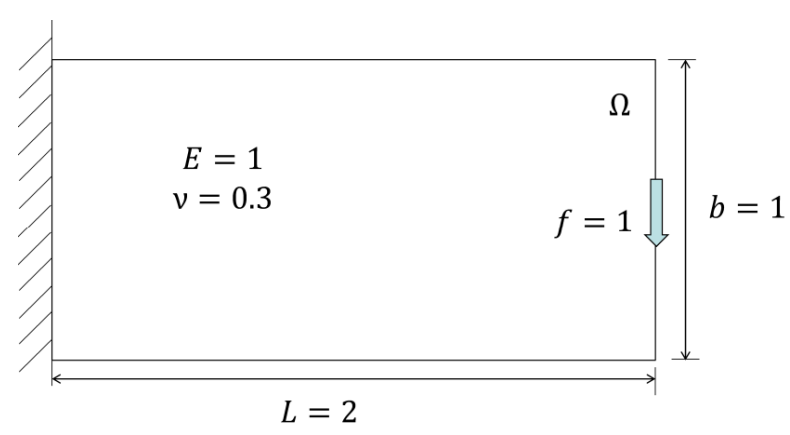

Fig. 1. Design problem description

\begin{tabular}{|c|c|}
\hline 0 & 0.5 \\
\hline 0 & \\
\hline 0 & 0.5 \\
\hline 0 & \\
\hline
\end{tabular}

Fig. 2. Failure probability distribution result

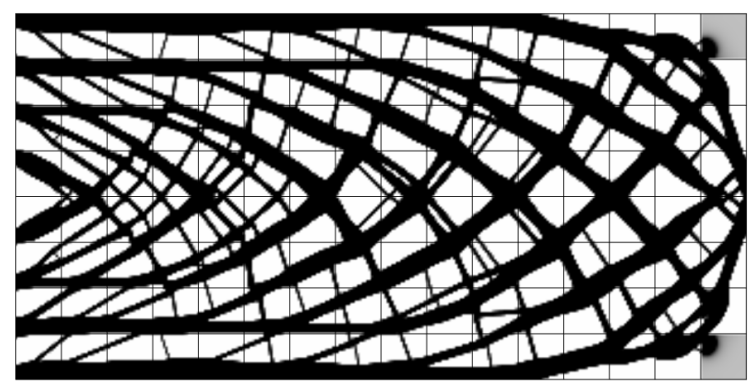

Fig. 3. Optimal robust structure in game situation

Holmberg, E., Thore, C.J., and Klarbring, A. (2017). Game theory approach to robust topology optimization with uncertain loading. Structural and Multidisciplinary Optimization, 55(4), 1383-1397.

Kawamoto, A., Matsumori, T., Yamasaki, S., Nomura, T., Kondoh, T., and Nishiwaki, S. (2011). Heaviside projection based topology optimization by a pde-filtered scalar function. Structural and Multidisciplinary Optimization, 44(1), 19-24. doi:10.1007/s00158-010-0562-2.

Papadimitriou, C.H. (2007). The complexity of finding nash equilibria. In N. Nisan, T. Roughgarden, E. Tardos, and V.V. Vazirani (eds.), Algorithmic Game Theory, 2951. Cambridge University Press, New York, USA.

Rozvany, G., Zhou, M., and Birker, T. (1992). Generalized shape optimization without homogenization. Structural optimization, 4(3), 250-252. doi:10.1007/BF01742754.

Tromme, E., Kawamoto, A., and Guest, J.K. (2017). Multiscale topology optimization based on reduction methods for additive manufacturing. Structural and Multidisciplinary Optimization, To be submitted.

Wu, J., Aage, N., Westermann, R., and Sigmund, O. (2017). Infill optimization for additive manufacturing - approaching bone-like porous structures. IEEE Transactions on Visualization and Computer Graphics, PP(99), 1-1. doi:10.1109/TVCG.2017.2655523. 